\section{OTU-020 ALTERED FC AND FAB GLYCOSYLATION STATUS IN PATIENTS WITH IGG4-RELATED SCLEROSING CHOLANGITIS AND AUTOIMMUNE PANCREATITIS}

${ }^{1}$ Emma Culver*, ${ }^{2}$ Fleur Sanne van de Bovenkamp, ${ }^{2}$ Ninotska Derksen, ${ }^{1}$ Tamsin Cargill, ${ }^{3}$ Carolien Koeleman, ${ }^{3}$ Lisa Neef, ${ }^{1}$ Eleanor Barnes, ${ }^{2}$ Rob Aalberse, ${ }^{3}$ Manfred Wuhrer, ${ }^{2}$ Theo Rispens. ${ }^{1}$ Translational Gastroenterology Unit, John Radcliffe Hospital and Nuffield Department of Medicine, University of Oxford, Oxford, UK; ${ }^{2}$ Sanquin Research, Department of Immunopathology and Landsteiner Laboratory, Academic Medical Centre, University of Amsterdam, Amsterdam, The Netherlands; ${ }^{3}$ Center for Proteomics and Metabolomics, Leiden University Medical Center, Leiden, The Netherlands

\subsection{6/gutjnl-2018-BSGAbstracts.306}

Introduction IgG4-related disease (IgG4-RD) is a systemic fibro-inflammatory condition characterised by an abundance of $\mathrm{IgG}^{+}$antibodies in the serum and tissue of involved organs. IgG glycosylation plays an important role in many chronic inflammatory and autoimmune conditions. We sought to assess the glycosylation status in patients with IgG4-RD and correlate with disease activity, damage and response to treatment.

Methods IgG Fc and Fab glycosylation status was assessed in patients with IgG4-RD involving the bile ducts (IgG4-sclerosing cholangitis, IgG4-SC) and pancreas (autoimmune pancreatitis) $(n=22)$, disease controls with primary sclerosing cholangitis (DC $n=22$ ) and healthy controls (HC $n=22)$. Disease activity, organ damage and response to treatment were assessed serially using the IgG4-responder index. Serum IgG and subclasses were quantified using an ELISA and nephelometry. IgG and subclass Fc glycosylation was analysed by mass spectrometry and Fab glycosylation by lectin (SNA) affinity chromatography. Statistics were performed using Prism.

Results IgG4-SC and AIP patients exhibited reduced total IgG Fc galactosylation and IgG1 Fc bisection, and increased IgG4 Fc fucosylation and IgG2/3 Fc hybrid compared with HC. There was recovery of IgG1 Fc bisection (increase) and IgG2/ $3 \mathrm{Fc}$ hybrid (decrease) upon corticosteroid treatment. IgG Fc galactosylation and $\operatorname{IgG} 2 / 3 \mathrm{Fc}$ hybrid correlated with disease activity. IgG Fab glycosylation was higher in IgG4-RD patients, with an increase in IgG4-specific, and to a lesser extent IgG1specific, Fab glycosylation compared to HC and DC.

Conclusions In the first study to assess glycosylation status in IgG4-RD, we demonstrated alterations in both $\operatorname{IgG~} \mathrm{Fc}$ and Fab glycosylation, which may play a role in pathophysiology and serve as a biomarker of disease.

\section{PTU-022 WHAT IS THE YIELD AND CLINICAL UTILITY OF EUS IN PATIENTS WITH PRIOR NON-DIAGNOSTIC MRCP?}

Noor Bekkali*, Fahd Rana, Manu Nayar, John Leeds, Kofi Oppong. Freeman Hospital, Newcastle, UK

\subsection{6/gutjnl-2018-BSGAbstracts.307}

Introduction The most effective investigation for suspected gallstones between MRCP and EUS is unclear. A 2015 Cochrane systematic review of their performance in common bile duct (CBD) stones concluded that the tests were of comparable accuracy. Conversely, a 2017 meta-analysis found EUS to be more sensitive. Any superiority of EUS may be due to better accuracy in detecting small stones. MRCP is routinely favoured as the 2nd line test following a non-diagnostic abdominal ultrasound and EUS subsequently performed as the 3rd line test when suspicion remains after a non-diagnostic MRCP. The yield and clinical utility of EUS in this setting is unclear. The aim was to identify the yield of EUS in patients with prior non-diagnostic MRCP undergoing EUS in our tertiary service.

Methods All EUS reports from 2017 were reviewed along with the electronic patient records to identify cases with prior MRCP. Indication for the procedure, symptoms, liver blood tests and interval between MRCP and EUS were recorded. Findings of sludge, microlithiasis (stones $<2 \mathrm{~mm}$ ) and discrete stones were categorised together as stones. Subsequent ERCP or cholecystectomy was identified. Yield was defined as a finding that would lead to a change in management.

Results A total of 1058 diagnostic EUS were screened of whom 253 (24\%) had prior MRCP and formed the study group. Median age was 58 (16-88) years, 179 (71\%) were female and $91(36 \%)$ had a cholecystectomy. Median interval between EUS and MRCP was $5.2(0.1-37)$ months. Indications for EUS were: $n=76(30 \%)$ dilated CBD, $n=65(26 \%)$ query CBD stones, $\mathrm{n}=54(21 \%)$ unexplained acute pancreatitis (AP), $n=23(9 \%)$ right upper quadrant pain, $n=17 \quad(6.7 \%)$ abnormal LFTs, $n=16(6.3 \%)$ double duct sign and $n=2(1 \%)$ dilated PD. There was a yield from EUS in $30(12 \%)$ patients with no significant difference between those with $(n=11)$ or without cholecystectomy $(n=19)$. Stones were identified in 24 cases with median size of $4 \mathrm{~mm}$ (range $2-8)$ in: CBD $(n=16)$, cystic duct $(n=1)$ and $\mathrm{GB}(\mathrm{n}=7)$. Three had abnormal CBD without stones (calcification $\mathrm{CBD}$ wall, thick walled $\mathrm{CBD}$, polyp), 1 patient with possible stone on MRCP had no stone seen on EUS, 1 had a pancreatic mass, and 1 had chronic pancreatitis. All patients in whom EUS findings indicated an intervention (26/30) have been referred: ERCP in 13, cholecystectomy in 9, ERCP and cholecystectomy in 3 and chemotherapy in 1 .

Conclusion EUS following non-diagnostic MRCP is a sizeable workload accounting for $24 \%$ of diagnostic activity in our unit with a clinically significant yield in $12 \%$ of predominantly small stones. Further prospective studies are required to ascertain the most cost-effective way to incorporate EUS into the investigation of suspected gallstone disease.

\section{PTU-023 ENDOSCOPIC ULTRASOUND BIOPSY PRIOR TO PALLIATIVE TREATMENT FOR PANCREATIC CANCER: CAN WE PREVENT UNNECESSARY PROCEDURES?}

Mustafa Jalal*, Jonathan Wadsley, Andrew Hopper. Gastroenterology department and Weston Park Hospital, Sheffield Teaching Hospitals, Sheffield, UK

\subsection{6/gutjnl-2018-BSGAbstracts.308}

Introduction Pancreatic adenocarcinoma (PDAC) has a very poor prognosis with most patients presenting with advanced incurable disease. Palliative chemotherapy can have a significant improvement in survival, but given the potential severe complications patient assessment and histological confirmation with endoscopic ultrasound fine needle aspiration (EUS-FNA) is required. The rapid progression of PDAC can result in patients urgently travelling to tertiary centres and undergoing EUS-FNA (which is an invasive, sedated procedure with associated morbidity) prior to formal assessment in patients where the chemotherapy is subsequently not given. We aimed to see if there are pre-test prediction factors for non-uptake of palliative chemotherapy in PDAC in our cancer network.

Methods We retrospectively reviewed consecutive patients referred for EUS-FNA over a 2 year period for evaluation of 
inoperable locally advanced pancreatic masses on imaging. Details recorded were: age, body mass index (BMI), co-morbidity including cardiovascular, diabetes mellitus, chronic airway disease and anticoagulation. Also recorded were the World Health Organisation (WHO) performance status, position of the tumour and the requirement of a biliary stent. Patients who received chemotherapy were identified from the chemotherapy registry data. The diagnosis of PDAC was based on histological diagnosis or with clinical progression compatible with the diagnosis or death from malignancy.

Results In total 104 underwent EUS-FNA [55 men, mean age 68.5 years SD \pm 9.1$]$. All patients had a performance status of $<3$. Of these patients $50(48.1 \%)$ went onto start palliative chemotherapy. None of the 8 patients $\geq 80$ years old who underwent EUS-FNA received chemotherapy compared to 50 out of $96(52.1 \%)$ patients $<80$ years old $(p=0.0014)$. There were no other significant differences or predictors of chemotherapy uptake in patients when analysing presence of comorbidity, position of tumour, jaundice at presentation, WHO performance status and BMI $<20$.

Conclusion In this study, no patients over 80 years old having undergone EUS-FNA went on to receive palliative chemotherapy for PDAC. We would advise an initial oncology consultation first in these patients to avoid unnecessary EUS procedures. In patients under 80 years old clinical assessment should be considered when referring patients for suspected inoperable PDAC for EUS-guided FNA as only half go on to receive treatment, however no factors apart form age seemed to predict the uptake of palliative chemotherapy. Further validation of these outcomes could form a decision tool to decide who should be triaged to oncology clinics before EUS-FNA performed.

\section{PTU-024 PANCREATIC CYSTS - CAN INVESTIGATIONS BE SAFELY RATIONALISED?}

Claire Deakin*, Gillian Flynn, Kate Wheeler, Pete Thurley, Imran Bhatti, Andrew Austin, Adam Lawson. Derby Teaching Hospitals NHS Foundation Trust, Derby, UK

\subsection{6/gutjnl-2018-BSGAbstracts.309}

Introduction Pancreatic cysts are a frequent incidental finding on cross-sectional imaging of the abdomen. We examined the decision making and outcome of patients with a pancreatic cyst(s) discussed at the Royal Derby Hospital HPB cancer MDT and compared practice against the 2015 American Gastroenterology Association (AGA) and 2017 International Association of Pancreatology (IAP) guidelines on the management of pancreatic cysts.

Methods A search of HPB MDT meeting reports, from January 2016 to October $2017(\mathrm{n}=1144)$ identified 88 patient $(51$ female) reports relating to the first discussion of a pancreatic cyst. Electronic medical records were examined to collect data pertaining to subsequent investigations and outcome. Details of medical comorbidities were used to calculate a Charleson comorbidity index.

Results The median age was 72 years (range 32-87) and the median estimated 10 year survival based on the Charleson Comorbidity Index was 53\% (range 0\%-98\%).

$86 \%$ of pancreatic cysts were judged to be an incidental finding. The median cyst diameter was $19.5 \mathrm{~mm}$ (range 4$110 \mathrm{~mm}) .43 / 88(49 \%)$ patients proceeded to endoscopic ultrasound (EUS), with 33 having a fine needle aspiration
(FNA). 4/88 (5\%) patients had probably malignant (C4) or malignant (C5) cytology. All 4 patients had "high risk stigmata" on their initial CT/MRI. The final outcome for most patients was no further intervention $(56 \%)$ or follow-up imaging (36\%), with $5(6 \%)$ patients offered surgery.

Applying the 2017 IAP management algorithm, 13 (15\%) patients had "high-risk stigmata" on CT/MRI and except where their performance status or co-morbidity precluded further investigation/treatment (5), were recommended for surgery (1) or EUS (7). Of the remaining 75 patients, 45 (60\%) had no worrisome features on CT/MRI and so would not have required EUS. 21/45 (47\%) of these patients in our practice underwent EUS, but none demonstrated definite mural nodules, main duct involvement or suspicious/positive cytology.

The 2015 AGA management algorithm could only be applied to those patients who had undergone initial radiological assessment with MRI $(n=11)$. None of these patients had two positive features on MRI, indicating a need for EUS. 6/ 11 (55\%) patients did, however, have an EUS, with none identifying positive features or concerning cytology.

Conclusions These findings suggest that a significant proportion (24\%) of patients with pancreatic cysts underwent unnecessary EUS. Application of international guidelines can reduce the number of patients who require an endoscopic ultrasound.

\section{PTU-025 EUS FNA MICROCORE BIOPSY IS SUPERIOR TO ENDOBILIARY BIOPSY IN DIAGNOSING MALIGNANT PANCREATICOBILIARY LESIONS}

ReeNee Tiam*, Ban Jalil, Justin Cooke. Hull and East Yorkshire Hospitals NHS Trust, Hull, UK

\subsection{6/gutjnl-2018-BSGAbstracts.310}

Introduction The ability to provide definitive and timely histological diagnosis of malignancy in suspected malignant pancreaticobiliary lesions depends on high quality tissue sampling. We conducted a study to evaluate the diagnostic utility of endobiliary biopsy versus endoscopic ultrasonography-guided fine needle aspiration (EUS FNA) microcore biopsy.

Methods We performed a retrospective search of our laboratory information management system for patients with clinically suspected malignant pancreaticobiliary lesions who had endobiliary and EUS FNA microcore biopsies. The haematoxylin and eosin-stained slides were retrieved and reviewed. All biopsies were categorised into whether a definitive diagnosis can be established (diagnostic) or not (non-diagnostic).

Results The search yielded 94 endobiliary biopsies and 78 EUS FNA microcore biopsies. 77 out of the 94 endobiliary biopsies were deemed adequate, and out of this 54 was diagnostic of malignancy (sensitivity 57\%). In 11 cases where the endobiliary biopsy was not diagnostic, subsequent EUS FNA microcore biopsies provided a malignant diagnosis in $9.96 \%$ of EUS FNA microcore biopsies were adequate and in 62 a malignant diagnosis could be established (sensitivity 83\%). Cholangiocarcinoma was the pathological diagnosis in the majority of the endobiliary biopsies and there were two metastases from lung and two neuroendocrine tumours. The main malignant diagnosis in the EUS FNA microcore biopsies was adenocarcinoma of pancreaticobiliary-type, but also included neuroendocrine tumours, solid pseudopapillary neoplasm and adenosquamous carcinoma. There were also three metastases from the colorectum, kidney and breast. 